\title{
Time Coupling Constraints Modeling and Analysis in Unit Commitment
}

\author{
Shuai Cao, Zhenglin Yang, Haihua Cheng, Yaxian Zheng and Jian Geng \\ China Electric Power Research Institute, Nanjing 210003, China
}

\begin{abstract}
Tight time coupling will require the high modeling performance, especially the minimum on/off time constraint. The authors referred to the thought of the cutting plane theory, and built 4 kinds of models ABCD of the minimum on/off time constraints, to completely demonstrate the problem. Then they gave 3 kinds of optimal modeling criterial, the solution accuracy, the node numbers and the peak memory. They clarified mathematically the model $C$ was the optimal to reduce the time couplings of coefficient matrix. Finally, IEEE118 case was used to validate the efficiency of the model and the criterial. With the characters of inequality to adjust the constraints, the guide method could be applied in other fields.
\end{abstract}

Keywords-time coupling constraints; unit commitment; cutting plane; optimal modeling criterion

\section{INTRODUCTION}

Unit commitment (UC) does research on determining the on/off states and powers of choosing units to satisfy the optimal objective during different time scales [2]. Actually it is a kind of the problem of mixed integer programming (MIP). UC problem is always being the hot spot for the advantage of economics and energy-saving in electrical automatic field.

The matrix dimension of UC problem grows up exponentially with the time periods. It makes the key point that how to build the most proper time coupling constraints, especially the minimum on/off time constraints, in the large time scale of UC. The branch and cut $(\mathrm{B} \& \mathrm{C})$ is one of the most popular methods in the main business software[3], and also the excellent model will improve the efficiency of B\&C. Nowadays there are several papers relative to the time coupling constraints modeling and analyzing in and out. The 3-binary states model in [4] was presented as the programming comparison, furthermore [5]imposed the modified 3-binarymodel, coupling the startup state and running state. However they were neither compared with other models. The 1-binary model formulated in [6]could reduce the number of integer variables and time constraints, and it was proved to have better quality than the 3-binarymodel [5]in saving the programming time and objective costs. But another case in [7]demonstrated the model of [5] was superior to [6]in node numbers. It needs some convincing optimal criteria. Also in [8], some optimal criteria which were summarized, nerveless it was lacked in systematic analysis. On the other hand, the application of cutting plane theory usually lied in the algorithm, rather than the guide for modeling procedure.

The objective of this paper is to establish the whole model systems of minimum on/off time constraints of the thermal unit commitment problem, hereinafter to choose the best model by the solution, the time and the space analysis. This paper refers the valid inequality and cutting plane, according to the actual scene, and models the minimum on/off time constraints. Meanwhile, with the characters of inequality to adjust the constraints, the guide method could be applied in other fields.

\section{Cutting Planes Theory}

Considering the MIP problem

$$
\min \left\{\mathbf{c}^{\mathrm{T}} \mathbf{x} \mid \mathbf{A x} \geq \mathbf{b}, \mathbf{x} \in \mathrm{N}^{n}+\mathrm{R}_{*}^{r}\right\}
$$

where $\mathbf{x}$ is a $n+r$ dimension column vector, representing $n$ dimension nature vector and rdimension non-zero real vector, $\mathbf{C}$ is the correlative column vector in objective function. $\mathbf{A}$ is a $k \times(n+r)$ dimension matrix, b presents $k$ dimension resource column vector, where $k$ is the order of maximal linear independent group.

The corresponding relaxing linear problem

$$
\min \left\{\mathbf{c}^{\mathrm{T}} \mathbf{x} \mid \mathbf{A} \mathbf{x} \geq \mathbf{b}, \mathbf{x} \in \mathrm{R}_{*}^{n}+\mathrm{R}_{*}^{r}\right\}
$$

The convex set of the relaxing linear problem

$$
\mathrm{P}_{\mathrm{R}}=\left\{\mathbf{x} \mid \mathbf{A x} \geq \mathbf{b}, \mathbf{x} \in \mathrm{R}_{*}^{n+r}\right\}
$$

The convex set of the MIP problem

$$
\mathrm{P}=\mathrm{P}_{\mathrm{R}} \mathrm{I}\left\{\mathrm{N}^{n}+\mathrm{R}_{*}^{r}\right\}
$$

Definition1 [9]The inequality $\mathbf{a}^{\mathrm{T}} \mathbf{x} \geq b$ is a valid inequality if $\mathbf{a}^{\mathrm{T}} \mathbf{x} \geq b$ for all $\mathbf{x} \in \mathrm{P}$.

According to Def.1, all inequalities in the constraints set of MIP are valid inequalities.

Definition2 [9]The valid inequality $\mathbf{a}^{\mathrm{T}} \mathbf{x} \geq b$ is a cutting plane if $\mathbf{a}^{\mathrm{T}} \mathbf{x} \geq b$ for $\mathrm{P}_{\mathrm{R}} \mathrm{I}\left\{\mathbf{x} \mid \mathbf{a}^{\mathrm{T}} \mathbf{x} \geq b\right\} \subset \mathrm{P}_{\mathrm{R}}$.

In other words, a cutting plane is a valid inequality which could just delete the wrong relaxing solutions from set $P_{R}$, doing nothing for feasible solutions in set $\mathrm{P}$. The two 
characteristics above are not only the essence of cutting planes, but also the basis of large-scale linear programming order reduction algorithm and linear programming relaxation technique [10].When using this method to solve the problem of MIP, as long as the linear relaxation solution doesn't meet $\left\{\mathrm{N}^{n} \times \mathrm{R}_{*}^{r}\right\}$, a cutting plane will be added. And then the new problem will be solve once again. It repeat the same process until meet the demand.

Experience shows that in in practical use of B\&C method, some preprocessing for the constraint set could improve the efficiency of computation [11]. Because the compact constraint set maydirectly make up the cutting plane, and especially if it is a convex cutting plane, the initial boundary could be much tighter. The boundary in this article is similar with the polyhedral theory in [8].The key variablesare binary in the UC problem of power system, which represent the states of the units. Due to what after the relaxation binary variables are just within the range of $[0,1]$, and it is good for the formation of initial cutting plane. Considering magnification and shrinking of inequalities, we can easily get the most compact constraints, and it can be proved extremely easily theoretically.

\section{Time Coupling Constraints Modeling}

The optimization objective of UC is usually the total cost of system or the lowest fuel consumption, the constraint conditions including system power balancing, reserve constraints, generation limits and ramping constraints, minimum on and off time constraints, etc. [11].

The UC model of MIP can be expressed by the symbolic form below.

$$
\min \left\{\mathbf{c}^{\mathrm{T}} \mathbf{x} \mid \mathbf{A x} \geq(=) \mathbf{b}, \mathbf{x} \in \mathrm{N}^{n}+\mathrm{R}_{*}^{r}\right\}
$$

This paper is focused on minimum on/off time constraints. In accordance with the time in a different direction, minimum on/off time constraints modeling method has been divided into the forward mode [5, 8], the backward mode [4, $6]$ and the bilateral mode [8,9].According to the number of state variables of units, there are two kinds of modeling ideas, that the 1-binary mode[6,8,9] and 3-binary [4, 5, 7] two kinds of modeling ideas.

In the actual operation of power system, at the end of the optimization period, generally there is no need to meet at least one operation cycle or outage cycle, and the bilateral mode may be slow in converging, so only the backward mode is advisable. In the condition of being different in constrained variables and thresholds, this paper established 4 kinds of models, covering all existing modeling methods, respectively named the $\mathrm{ABCD}$, as shown in Fig. 1 below. o describe the unit operation of 3-binary, $u$ is the unit running state, $\mathrm{y}$ is unit start-up state, $\mathrm{z}$ is the shut-down state.

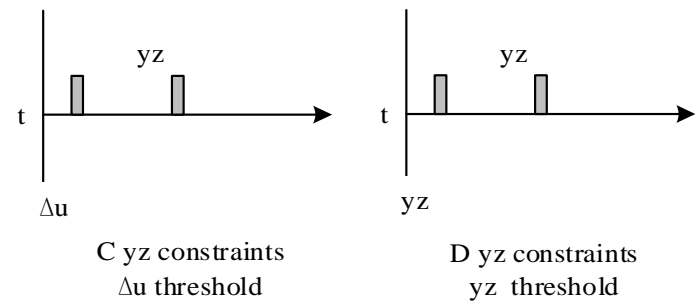

FIGURE I. FOUR KINDS OF MINIMUM ON/OFF TIME CONSTRAINTS MODELS

Mode A can be formulated as

$$
\left\{\begin{array}{c}
\sum_{\tau=t+1}^{\min \{t+M U T-1, T\}} u_{i \tau}-\left(u_{i t}-u_{i(t-1)}\right) \geq 0 \\
\sum_{\tau=t+1}^{\min \{t+M D T-1, T\}} 1-u_{i \tau}-\left(u_{i(t-1)}-u_{i t}\right) \geq 0
\end{array}\right.
$$

Model A means that after the start-up (or shut-down), units must remain the on(or off)states for at least the minimum period. The unit states of a time period will be limited strictly by the first time segment. Among them, MUT represents the minimum on time, $M D T$ represents the minimum off time. Where $u_{i \tau}$ is the restricted variable, $\Delta u_{i t}$ is the bound variable. Start segment is qualified naturally.

$$
\left\{\begin{array}{l}
u_{i \tau} \geq u_{i t}-u_{i(t-1)}, \tau=t+1, \ldots, \min \{t+M U T-1, T\} \\
1-u_{i \tau} \geq u_{i(t-1)}-u_{i t}, \tau=t+1, \ldots, \min \{t+M D T-1, T\}
\end{array}\right.
$$

Eq. 6 is obtained by linear superposition of Eq. 7. And according to the theory of linear programming, the linear superposition of the same directional valid inequality can increase feasible regions, even if rate increasing is not high. But Eq. 6 is obviously better than the Eq. 7. Compared to Eq. 7, the number of independent constraints Eq. 6 decreases at least by $50 \%$, and the ideas of the essence of which is the combination of the initial cut and decoupling. Finally, the selected model A is Eq. 6.

Mode B can be formulated as

$$
\left\{\begin{array}{c}
\sum_{\tau=t+1}^{\min \{t+M U T-1, T\}}\left(u_{i \tau}-y_{i t}\right) \geq 0 \\
\sum_{\tau=t+1}^{\min \{t+M D T-1, T\}}\left(1-u_{i \tau}-z_{i t}\right) \geq 0
\end{array}\right.
$$

Model B have the same constrained variables as model $\mathrm{A}$, but the $\mathrm{y}$ and $\mathrm{z}$ threshold. The $\mathrm{yz}$ represent the difference of state, and in other words, model B is actually a set of first-order state inequality. By introducing linear 
logic of constraint Eq. 9 of yz, the threshold variable of model $\mathrm{B}$ is bigger than the A. Considering the inequality direction, constrained variables are in enhanced limits, and the linear relaxation of the feasible region become smaller, but the MIP feasible region does not change. Therefore the valid inequalities of model $B$ can be seen as the initial cutting plane. In theory, the effect of the model B is better than A. However it is also should be cautious that for the introduction of independent integer variables, the model B may show the optimal performance after a certain time period.

$$
\left\{\begin{array}{l}
u_{i t}-u_{i(t-1)}=y_{i t}-z_{i t} \\
y_{i t}+z_{i t} \leq 1
\end{array}\right.
$$

For model C as Eq. 10, constrained variables are yz, and $\mathrm{u}$ is threshold. Model $\mathrm{C}$ has the different modeling routine, focused on the change of state variables, rather than state variables. Hence the amount of effective limited constraint is larger than model A or B, but limited variables in each constraint are better in quantity.

$$
\left\{\begin{array}{c}
\sum_{\tau=t+1}^{\min \{t+M D T-1, T\}} y_{i \tau} \leq 1-u_{i t} \\
\sum_{\tau=t+1}^{\min \{t+M U T-1, T\}} z_{i \tau} \leq u_{i t}
\end{array}\right.
$$

Model D can be formulated as

$$
\left\{\begin{array}{c}
\sum_{\tau=t+1}^{\min \{t+M D T-1, T\}} y_{i \tau} \leq 1-z_{i t} \\
\sum_{\tau=t+1}^{\min \{t+M U T-1, T\}} z_{i t} \leq 1-y_{i t}
\end{array}\right.
$$

Model D is the often used as constraints of the form [4], and yz are both constrained and threshold. The amount of effective limited constraint is less, and also limited variables in each constraint are better. But the coupling degree of model D is higher than all models above.

\section{NUMERICAL RESULTS}

The proposed formulation has been applied to solve the IEEE118 data case[4]. All models have been implemented on a Lenovo Desktop with Corei5-4590 @ $3.30 \mathrm{GHz}$ and 8 GB of RAM memory using CPLEX 12.6. In this case study, the execution tolerance of CPLEX was within $1 \%$ of the optimal solution.

The test models were named ABCD, and the optimization cycle is $24 * 1 \mathrm{~h}$, expanding different time scale to contrast the model performance. Compared events included the characteristics of the solution (objective values, gap), time performance (computing time, number of nodes), and space performance(peak memory).The initial states of thermal units are fully on and the initial power is the minimum output. The system reserve under highest load remains $22.5 \%$.

\begin{tabular}{|c|c|c|c|c|c|c|c|c|c|}
\hline \multirow{2}{*}{ Period/h } & \multicolumn{4}{|c|}{ Generation cost higher proportion (\%) } & \multirow{2}{*}{ Period/h } & \multicolumn{4}{|c|}{ Optimal power generation costs (k\$) } \\
\hline & A & $\mathrm{B}$ & $\mathrm{C}$ & $\mathrm{D}$ & & A & $\mathrm{B}$ & $\mathrm{C}$ & $\mathrm{D}$ \\
\hline 24 & $0.27 \%$ & $0.26 \%$ & $0 \%$ & $0.26 \%$ & 24 & 1351.74 & 1351.66 & 1348.15 & 1351.66 \\
\hline 48 & $0.25 \%$ & $0.78 \%$ & $0 \%$ & $0.27 \%$ & 48 & 2702.05 & 2716.28 & 2695.23 & 2702.63 \\
\hline 72 & $0.43 \%$ & $0.60 \%$ & $0 \%$ & $0.22 \%$ & 72 & 4059.56 & 4066.73 & 4042.31 & 4051.33 \\
\hline 120 & $0.19 \%$ & $0.75 \%$ & $0 \%$ & $0.18 \%$ & 120 & 6749.06 & 6787.33 & 6736.47 & 6748.45 \\
\hline 168 & $0.23 \%$ & $0.24 \%$ & $0 \%$ & $0.17 \%$ & 168 & 9452.74 & 9453.55 & 9430.63 & 9447.05 \\
\hline 360 & $0.31 \%$ & $0.26 \%$ & $0 \%$ & $0.17 \%$ & 360 & 20268.92 & 20260.38 & 20207.26 & 20242.32 \\
\hline 720 & $0.30 \%$ & $0.15 \%$ & $0 \%$ & $0.17 \%$ & 720 & 40536.70 & 40475.45 & 40413.46 & 40481.15 \\
\hline
\end{tabular}

TABLE I. THE COMPARE OF DIFFERENT MODELS

TABLE II. THE OPTIMAL RESULTS OF DIFFERENT MODELS

The correctness of the objective value is most important. Based on the minimum cost value of every time period, the objective values of different models are much closer, among which the highest proportion is $0.78 \%$, as shown in Table 1.With the period expanding, objective costs almost grow linearly, as shown in Table 2. In conclusion, each model almost has the similar objective value.

Then the accuracy of various models will be analyzed. Gap is the gap percent between linear relaxed solution and the optimal solution, as well as the convergence criterion. Because UC problem is NP-hard problem, and it is almost impossible to get the real optimal solution. So the Gap becomes the direct indicator of the precision in the solution. The Gap of each model is shown in Fig.2.Under the same convergence, Gap of model $\mathrm{C}$ is the most stable one, always staying below $0.01 \%$. The second is the model $\mathrm{D}$, below 0.1\%.Model A and B are inferior to CD, which means the initial cutting of the state change is better than simple status. Model A has been fluctuant in some top periods, while later it becomes smooth. For the similar modeling idea, model $\mathrm{B}$ is the same. However in the long period of time, model B is more advantageous to model A. In conclusion, the model $\mathrm{C}$ has the most solution characteristics among 4 kinds of models above, namely optimal feasible domain. 


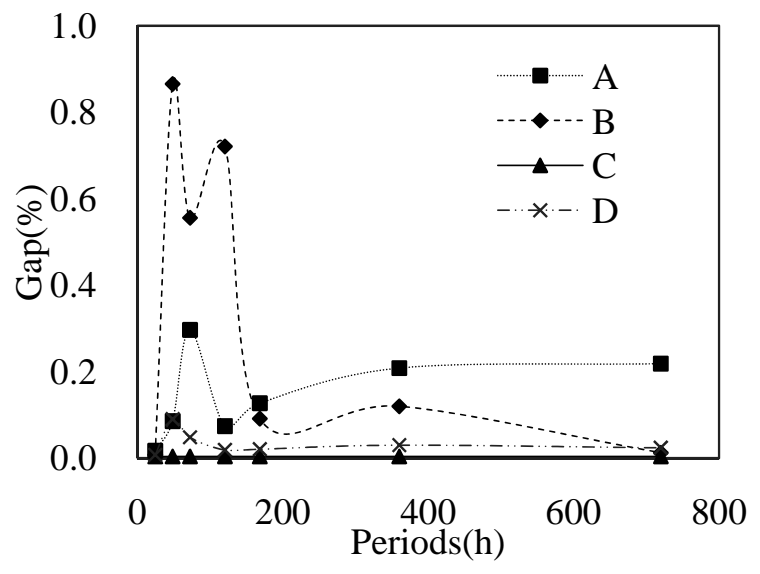

FIGURE II. THE GAP OF DIFFERENT MODELS

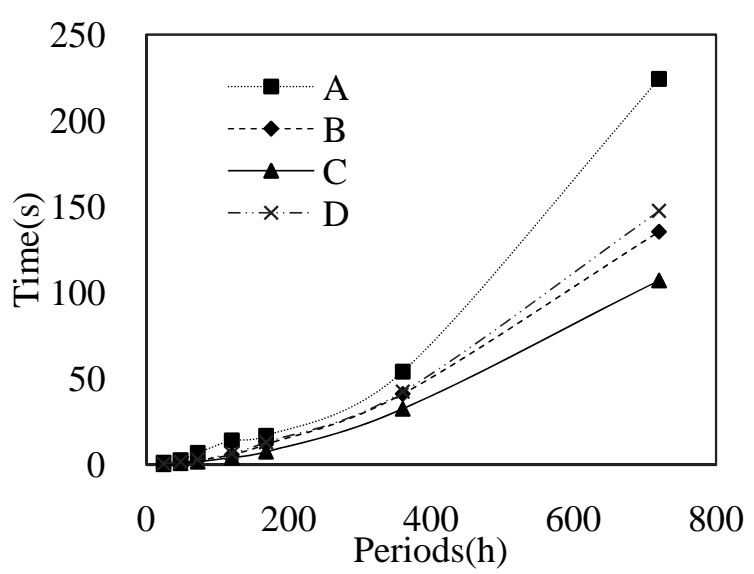

FIGURE III. THE COMPUTING TIME OF DIFFERENT MODELS

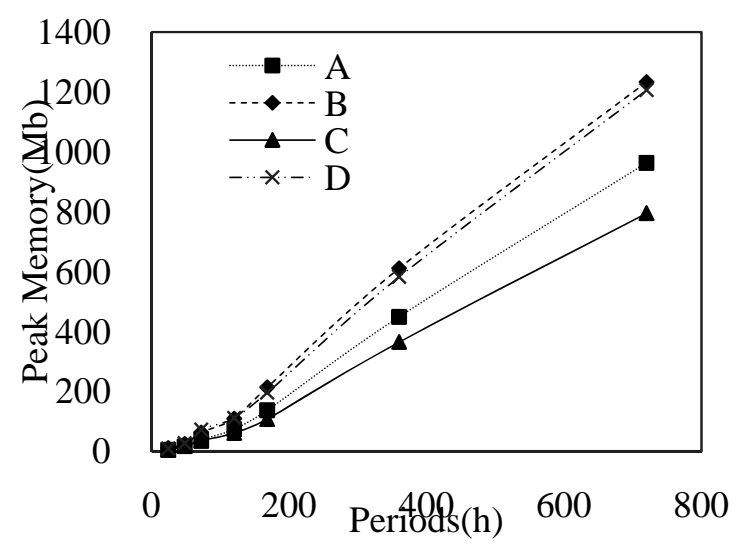

FIGURE IV. THE PEAK MEMORY OF DIFFERENT MODELS

Computing time is one of the most important indicators of UC. There is a positive correlation between computing time and the time scale. If entering into the branch procedure, there is a positive correlation between computing time and the number of effective branch nodes. Whether entering into the branch or not depends on the initial upper bound, and the convergence precision, but it can be increased by improving the initial cut to decrease the invalid branch probability, especially improving the root node. Under the convergence of $1 \%$, the model B, C, and $\mathrm{D}$ have not been in branch, while the model A has 56 and 110 nodes respectively in the period of $72 \mathrm{~h}$ and $120 \mathrm{~h}$, which results in computing time significantly enlarging. According to Fig. 3, the computing time of model $\mathrm{C}$ is the least, and it effectively reduces the dimension of the problem. Although model $\mathrm{D}$ also reduces the dimension problem, it strengthens coupling degree. Model A appears worst on time performance. At any time extension, model C's computing time grows upstably, nevertheless the model B and D rise fast, and model A fluctuates highly. Consequently, the best choice of the modeling should be the model C.

The peak memory is the representative index of space performance. The peak memory can reflect not only the model scale, but the peak memory used in model solving. As shown in Fig. 4, the peak memory of model $\mathrm{C}$ is considerably lower than the others. Also the peak of model $\mathrm{A}$ is less, because it has the least number of independent variables.

In consideration comprehensively of correctness, accuracy, time performance and space performance, model $\mathrm{C}$ should be the chose none. The advantage of model $\mathrm{A}$ is the small size of upper bound, but further dimension reduction is on low likelihood, which let alone the poor computing stability. Model B is in the middle position. Model D has one of the biggest scales, but the accuracy is second only to model C, so it can be used as a Plan B.

\section{SUMMARY}

The paper has referred to the ideas of the cutting plane, analyzing inequality variables of both sides to construct the initial cutting plane, and put forward 3 criteria to evaluate the initial cutting: the accuracy of the solution evaluating the refinement of feasible region, the efficiency node numbers evaluating the reduction of coefficient matrix dimension, and the peak memory usage evaluating the size of models. Under the actual operation, there are 4 classes to model the minimum on/off time constraints. Then the optimal model has been selected mathematically in theory. At last, numerical results have revealed the accurate and computationally efficient performance of the formulation.

The actual operation of power system is extraordinarily complicated, and it is necessary to test the model under changeable conditions. The degree of coupling still needs further research, such as analyzing the single correlation of coefficient matrix, the quantitative relationship between size limit and model performance, and so on.

\section{ACKNOWLEDGEMENT}

This research was financially supported by Science and Technology of SGCC(DZN17201500036).

\section{REFERENCES}

[1]. Xia Qing, Zhong Haiwang, Kang Chongqing, Review and Prospects of the Security Constrained Unit Commitment Theory and Applications, Proceedings of the CSEE, 2013, 33(16): 94-103. 
[2]. Zhang Meidan, Research on Optimal Operation of Electrical Power System, Zhejiang University, 2013.

[3]. N.P. Padhy, Unit Commitment-A Bibliographical Survey, IEEE Transactions on Power Systems, 2004, 19(2): 1196-1205.

[4]. Li Tao, M.Shahidehpour, Price-Based Unit Commitment: A Case of Lagrangian Relaxation Versus Mixed Integer Programming, IEEE Trans on Power Systems, 2005, 20(4): 2015-2025.

[5]. J M. Arroyo, A J. Conejo, Optimal Response of a Thermal Unit to an Electricity Spot Market, IEEE Trans on Power System, 2000, 15(3): 1098-1104.

[6]. M. Carrión, J.M. Arroyo, A Computationally Efficient Mixed-integer Linear Formulation for the Thermal Unit Commitment Problem, IEEE Transon Power Systems, 2006, 21(3): 1371-1378.

[7]. J. Ostrowski, M.F. Anjos, A. Vannelli, Tight Mixed Integer Linear Programming Formulations for the Unit Commitment Problem, IEEE Trans on Power Systems, 2012, 27(1): 39-46.

[8]. Xu Fan, Yao Jianguo, Geng Jian, Yang Zhenglin, Valid Inequality Analysis on Polytope of Unit Status in Unit Commitment, Power System Technology, 2011, 35(5): 82-89.

[9]. Zhang Lifu, Research on Valid Inequalities and Cutting Planes in Integer(mixed) Linear Programming, Xiangtan University, 2004.

[10]. IBM ILOG CPLEX V12.1 User's Manual for CPLEX, International Business Machines Corporation 1987, 2009: 501-510.

[11]. Geng Jian, Xu Fan , Yao Jianguo, Gao Zonghe, Ding Qia, Yang Zhenglin, Cheng Yun, Chen Gang, Performance Analysis of Mixed-integer Programming Based Algorithm for Security Constrained Unit Commitment, Automation of Electric Power Systems, 2009, 33(21): 24-27. 